\title{
Desafíos de la educación superior en el entorno contemporáneo
}

\author{
María Gabriela Ramos Barrera ${ }^{6}$
}

\section{Introducción}

ctualmente se le atribuye la escasa generación de conocimiento a la falta de inves-
tigación por parte de las universidades, dadas las fallas presupuestarias, falencias
administrativas en las instituciones, escasez de personal capacitado, o dependencia de grupos dominantes. Sin embargo, esta es solo una arista del problema y no se debe dejar de lado los escenarios en los cuales la educación superior le ha fallado a la sociedad por no crear un conocimiento pertinente, pues existe una separación entre el pensamiento académico y el contexto real en donde la formación se ha enfocado en repeticiones y procesos de enseñanza y aprendizaje que no se traducen en la aplicación práctica dentro de la sociedad.

Por ende, generar conocimiento pertinente que cumpla con las expectativas de las nuevas generaciones y de las nuevas dinámicas de la sociedad es fundamental para mantener la razón de ser de las instituciones de educación superior. En este sentido, es importante que se piense nuevamente el rol de la educación superior dentro de la sociedad, adaptándose a las realidades y contextos actuales, tomando en consideración los cambios tanto en los procesos de enseñanza como en los procesos de aprendizaje.

\section{Evolución de la educación superior: tiempos de adaptación}

La universidad es una institución fundamental para el desarrollo de las nuevas capacidades industriales y tecnológicas requeridas por las naciones, ya que contribuye en la formación de personas competentes con habilidades técnicas y administrativas que se requieren en

6. Profesora Asociada, adscrita al Programa de Negocios Internacionales de la Institución Universitaria Politécnico Grancolombiano, Bogotá-Colombia. Economista, con Maestría en Economía Cuantitativa de la Universidad de Los Andes, Mérida-Venezuela. Correo: HYPERLINK "mailto:mgramos@poligran.edu.co"mgramos@poligran. edu.co 
la sociedad moderna. Sin embargo, esta formación durante la mayor parte de la historia se consideró como una actividad exclusiva de los reyes, los nobles y la comunidad religiosa, y solo hasta finales del siglo XV la educación se consideró un espacio al que la mayor parte de la población podía acceder.

La tabla 5 resume brevemente la evolución de la educación superior, con el fin de comprender el rol que hoy tiene la educación dentro de la sociedad.

Tabla 5. Evolución de la Educación Superior

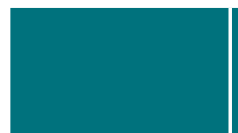

\section{Características principales}

$\begin{aligned} & \text { En el siglo V a.c. los griegos plantean la organización racional del conoci- } \\ & \text { miento con base en el orden de la realidad, mediante la cual representantes } \\ & \text { como Platón y Aristóteles dirigían a una minoría seleccionada para centrar } \\ & \text { los pensamientos en el conocimiento especulativo. }\end{aligned}$
La Biblioteca real de Alejandría (S. III a.c - S. IV d.c), representó una evo-
lución innovadora para la educación, porque creó el primer formato de estu-
dio sistemático en ciencias como matemática, física, biología, astronomía,
literatura, geografía y medicina que, adicionalmente, era gratuito, de libre
asociación, enfocado en el aprendizaje y con una dinámica plural (se pro-
movía la discrepancia en idiomas, creencias y pensamientos).

Fue una institución enfocada en el estudio de la filosofía y la religión donde, originalmente, la educación no se impartía de forma masiva, sino que el deber del maestro era desarrollar las cualidades potenciales de sus discípulos por medio de la relación personal.

Con la llegada del budismo se reorganizó la forma de impartir educación, se produjo un tránsito hacia la educación colectiva en la que los monasterios permitieron el ingreso de estudiantes laicos y, aunque la educación era gratuita, reyes y particulares ricos otorgaban subsidios en tierras que la universidad debía hacer rentables.

Sin embargo, la institución declinó cuando los estudios religiosos se desvincularon de las realidades sociales y se aislaron dentro de los monasterios. 
Características principales

Para el siglo XI las congregaciones religiosas organizaron escuelas para preparar a sus miembros en temas de teología y filosofía, y los reinados participaban para enseñar costumbres sofisticadas a los nobles. Estas universidades se caracterizaron por su sentido corporativo, pues no pagaban impuestos, tenían jurisdicciones especiales y poseían el derecho a protestar.

Por otro lado, los estudiantes elegían a sus profesores, se impartían clases magistrales y no existía un sistema de graduación sistemática. Se basaba en la discusión y no en la manipulación de objetos, por lo que se comienzan a dar quiebres entre lo práctico y lo teórico.

Con el inicio del capitalismo, el reconocimiento de las naciones y el establecimiento de las burguesías, la universidad medieval comenzó su declive, para dar entrada al acceso libre y directo a los libros (gracias a la invención de la imprenta a finales del siglo XV), y la Reforma protestante que cuestiona la existencia de una verdad única.
A partir de la consolidación del Estado laico, la educación comienza a orga- nizarse con base en los intereses del Estado, formando súbditos militares y funcionarios con características intelectuales y disciplinarias, que da origen a la educación pública estatal.
Luego, con la Revolución Francesa, los intereses se tornan a la forma- ción cívica y patriótica, para fomentar la participación crítica de los ciuda- danos en el gobierno, dando origen a la educación pública democrática con la participación de cualquier individuo, independientemente de su posición económica y social.

Fuente: elaboración propia con base en Carrera y Suayter (sf).

Se puede afirmar, entonces, que a lo largo de la historia el rol fundamental de la educación superior ha sido generar nuevos conocimientos que fomenten el crecimiento y bienestar de la sociedad, tomando como base una perspectiva interdisciplinar que considere aspectos de índole social, económico, político y cultural; y que el éxito de esta meta será determinado por el nivel de aplicación que tengan esos nuevos conocimientos dentro de la sociedad. Así, la evolución de la educación superior se ha generado producto de la adaptación de las instituciones a las dinámicas y contextos de la sociedad, sin embargo, estos procesos de adaptación se enfrentan a nuevos retos. 
En este sentido, en el año 1995, la Organización de las Naciones Unidas para la Educación, la Ciencia y la Cultura (Unesco, por sus siglas en inglés), en el Documento de Política para el cambio y el desarrollo de la Educación Superior, establece que el nuevo concepto de la educación superior se debe fundamentar sobre la base de tres pilares: pertinencia, calidad e internacionalización.

Respecto a la pertinencia, la Unesco establece el rol de la enseñanza superior como un sistema conformado por las instituciones y la sociedad, en donde la retroalimentación de ambos agentes es la esencia de la interacción. Es prioritario, entonces, que las instituciones de educación superior democraticen el acceso, se vinculen con los mercados laborales y tengan una relación permanente con el sistema educativo primario y secundario; y que, al mismo tiempo, la sociedad participe activamente dentro de los procesos de investigación de las universidades, con el fin de asegurar que las actividades académicas sean adecuadas para las realidades de la sociedad. De acuerdo con estas políticas para el cambio y el desarrollo:

Limitarse a la ampliación del contenido de los programas de estudio y al aumento del volumen de trabajo de los alumnos no puede ser una solución duradera. Han de considerarse, pues, prioritarias las materias que contribuyen al desarrollo de las capacidades intelectuales de los alumnos y les permiten adaptarse razonablemente a los cambios y la diversidad tecnológicos, económicos y culturales, que les infunden cualidades como el espíritu de iniciativa y de empresa o la capacidad de adaptación, y que les permiten desenvolverse mejor en un medio de trabajo moderno. (Unesco, 1995, pp. 30-31)

Respecto a la calidad, el documento de la Unesco enfatiza que este concepto es multidimensional, pues depende del sistema institucional, las características propias de cada disciplina y las posibilidades de aplicar los conocimientos creados pero que, a pesar de los diversos aspectos que contempla, debe referirse a "todas sus funciones y actividades principales: calidad de la enseñanza, de la formación y la investigación, lo que significa calidad del personal y de los programas, y calidad del aprendizaje, como corolario de la enseñanza y la investigación" (Unesco, 1995, p. 37).

Alcanzar la calidad implicaría, entonces, mejorar la eficacia de los docentes y de los contenidos programáticos, donde se reconozcan y promuevan las aptitudes para la enseñanza, la investigación y la formación para el perfeccionamiento profesional; mejorar las habilidades de los estudiantes en busca de una "movilidad social ascendente" o una "movilidad profesional horizontal", que permita la igualdad de oportunidades para cursar estudios superiores y desarrollar conocimientos por medio de métodos de aprendizaje eficientes; y mejorar la infraestructura y el entorno de las instituciones para garantizar no solo la modernización institucional, sino la integración con la sociedad al celebrar eventos (culturales, cívicos y deportivos), que permitan la interacción entre ambos agentes. 
Finalmente, respecto a la internacionalización, la Unesco establece que la educación superior es un reflejo del aprendizaje mundial que se fortalece en la medida en que se comprendan las interacciones interculturales, de los mercados y de las comunicaciones, y se incorporen los procesos de integración económica y política; por esto, la cooperación internacional debe ser una prioridad de los sistemas educativos. Es así como las nuevas tecnologías se convierten en facilitadoras de estos procesos de internacionalización, al promover las interacciones requeridas por las instituciones de una forma inmediata, efectiva y con menor costo.

Entonces, en este orden de ideas:

La búsqueda de soluciones para lograr que la educación superior alcance un mayor grado de pertinencia, calidad e internacionalización, exige que se vuelva a centrar la reflexión en el carácter central del desarrollo de los recursos humanos y del papel que desempeña la educación, en todos sus niveles y formas. (Unesco, 1995, p. 44)

\section{La estandarización de la educación superior en Colombia: del discurso a los indicadores}

De acuerdo con la Unesco, tener un nivel de educación superior se traduce en crecimiento de la sociedad, por lo tanto, que la población tenga acceso al sistema es prioritario. En este sentido, de acuerdo con cifras de la OCDE (2014), el sistema de educación de Colombia tiene un nivel mayor de estudiantes matriculados que el promedio de los países de la OCDE, y parte de esto se debe a la proliferación de instituciones privadas que ofrecen nuevos programas académicos, en donde se encuentra inscrita el 47\% de la población estudiantil (comparado con el 30\% promedio de la OCDE).

Incluso, respecto a la participación por género, se evidencian los avances en términos de igualdad, pues según las cifras de la OCDE, la participación de las mujeres dentro de la educación superior es significativa, incluso un poco mayor, pues en Colombia, para el año 2017, el 55\% de la población registrada dentro de las instituciones de educación superior correspondía a la femenina.

Sin embargo, la elevada participación de la educación privada implica, en un país con los altos niveles de desigualdad de Colombia, que el acceso a la educación es cada vez menos equitativo. Para mejorar estos niveles de participación, el Estado colombiano ha dedicado cerca del 15\% del gasto público al financiamiento del sistema de educación superior en el país, lo que sería equivalente al 4,4\% del PIB, durante los últimos doce años. Sin embargo, como evidencia el Gráfico 1, las proporciones se han mantenido relativamente constantes a pesar de las variaciones del PIB en Colombia. 


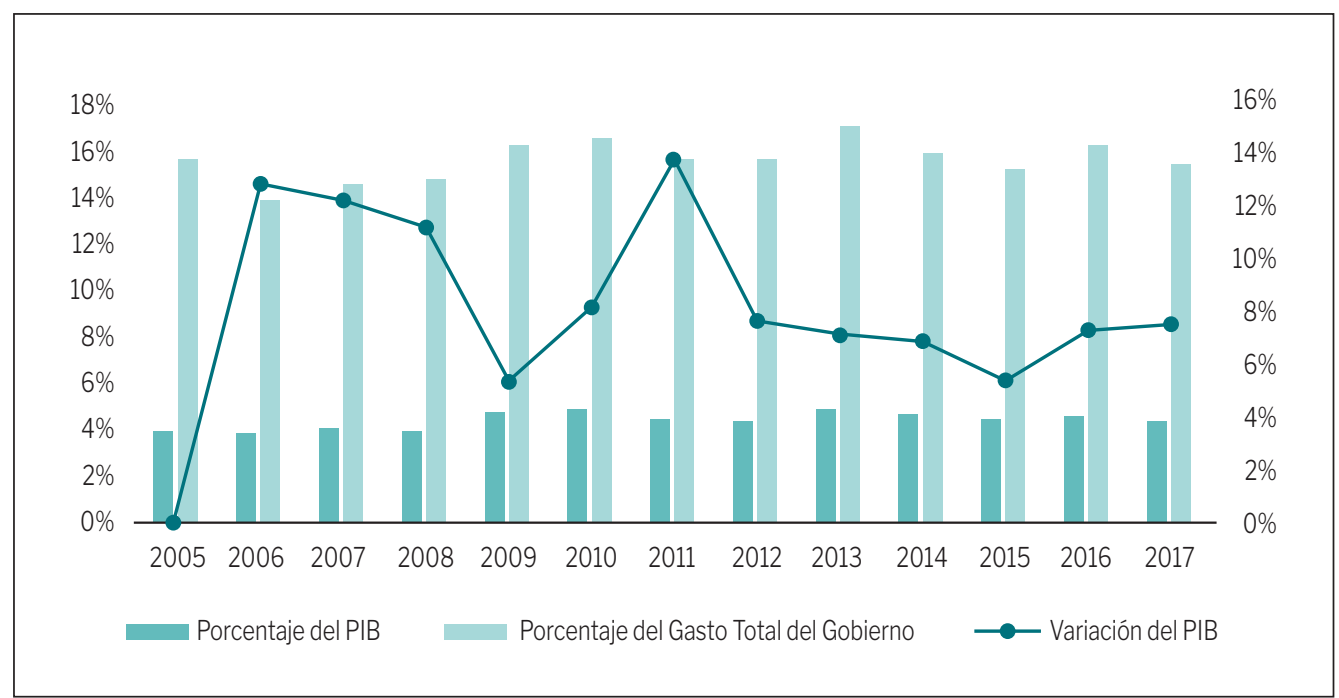

Fuente: elaboración propia con base en datos de la CEPAL (2018).

Nota: en el eje derecho del gráfico se leen las series de gasto en educación superior como porcentaje del PIB y como porcentaje del gasto total del gobierno. Al lado derecho del gráfico se lee la variación porcentual del PIB.

De este gasto, de acuerdo con cifras del Ministerio de Educación de Colombia, durante el año 2013, la ejecución total de la educación superior fue de USD 2000 millones, de los cuales $47 \%$ se ejecutaron en transferencias directas a las instituciones de educación superior, $20 \%$ se ejecutó mediante créditos estudiantiles con el Icetex, y el restante fue distribuido entre los diferentes organismos que fomentan la educación e investigación en el país, promedios que se mantuvieron en los períodos posteriores; pero no implicó mejoras en las condiciones de la educación pública.

Con respecto a esta asignación, uno de los principales hallazgos en la revisión de la OCDE y del Banco Mundial sobre la educación superior en Colombia es que "los sistemas de asignación de recursos públicos entre las instituciones de educación superior no están correctamente alineados con las necesidades económicas del país y las aspiraciones de los estudiantes". (OCDE, 2014, p. 271)

Por otro lado, además de la capacidad de generar conocimiento, que podría darse con una asignación eficiente de recursos, la importancia que genera la participación de la población dentro de la educación de tercer nivel se da dentro del nivel de remuneraciones salariales. El gráfico 2 muestra cómo la población que tiene educación terciaria tiene una mayor participación dentro del grupo de personas asalariadas que cuentan con ingresos de tiempo completo, a diferencia de aquellas personas que solo cuentan con niveles de formación primaria o secundaria. 
Gráfico 2. Porcentaje de la población entre 25 y 64 años, por nivel de educación y categoría de remuneración

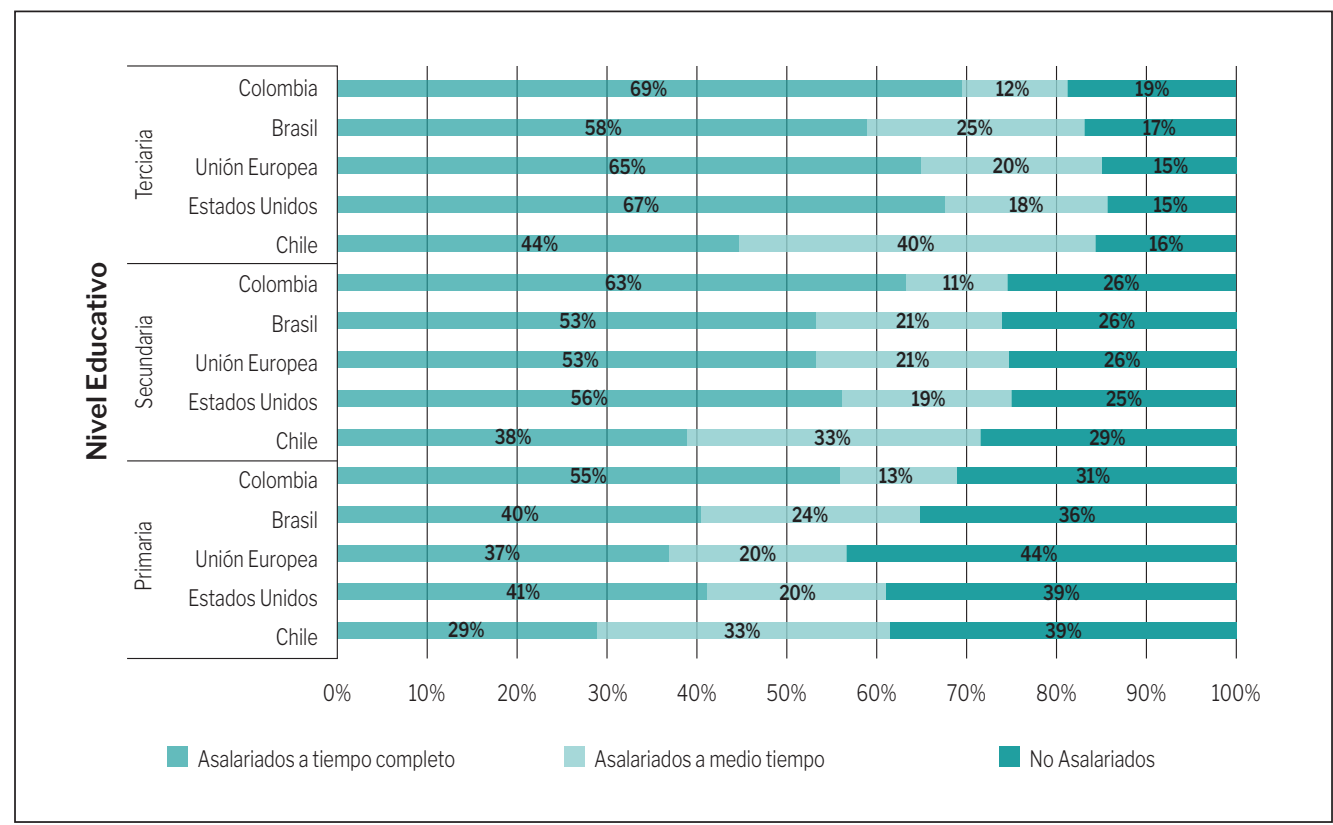

Fuente: Elaboración propia con base en las estadísticas de la OCDE (2018).

Nota: dentro de los países de la Unión Europea se contabilizan los 22 que forman parte de la OCDE.

En el caso de los países más desarrollados, como Estados Unidos o los miembros de la Unión Europea, se puede evidenciar claramente el valor que el mercado laboral le da a la educación terciaria, pues las personas que solo cuentan con estudios de primaria se encuentran en condición de no asalariados, o con remuneraciones de solo medio tiempo. En el caso de Estados Unidos, la diferencia es de 26 puntos porcentuales entre un nivel educativo de primaria y uno terciario, mientras que para los países de la Unión Europea esta diferencia se encuentra en 28 puntos porcentuales.

En países de la región, como Chile o Brasil, el comportamiento es relativamente similar, considerando que tener una titularidad de educación superior mejora las posibilidades de formar parte de un mercado laboral con remuneración de tiempo completo. Sin embargo, la diferencia dentro de esta mejor condición es menor en comparación con los casos anteriormente mencionados, pues en el caso de Chile es de 15 puntos porcentuales, mientras que para Brasil la diferencia es de 18 puntos.

Ahora, para el caso particular de Colombia es notorio que la diferencia en la categoría de remuneración es más estrecha, pues apenas es de 14 puntos porcentuales. Esto implica que, a pesar de tener acceso a la educación superior, obtener un grado académico de este 
nivel no necesariamente implica mejorar las condiciones laborales en término de las condiciones salariales.

La discusión se centraría, entonces, en explicar cómo mejores condiciones de educación superior incrementan la posibilidad de insertarse en un mercado laboral que brinde mejores condiciones salariales. Parte del problema se encuentra en la fuerte desvinculación que tienen las universidades con la sociedad y, especialmente, con respecto a las necesidades de las empresas (públicas o privadas) que son las generadoras de empleo.

A pesar de la participación de personas capacitadas, las universidades del país no están generando conocimientos que se vinculen de forma permanente con el sector productivo. Además de asegurar altos niveles de participación dentro del mercado laboral, la educación superior también debe garantizar que los procesos de aprendizaje y enseñanza sean capaces de transmitir nuevo conocimiento a la sociedad.

En este sentido, de acuerdo con Moreno y Ruiz (2009), debe existir una estrategia específica y clara en el largo plazo, donde exista comunicación entre el Estado y el sector privado para promover los procesos de innovación, que generen en América Latina espacios para el aumento de la productividad, para que la generación de conocimiento de las instituciones de educación superior pueda ser pertinente.

Sin embargo, cuando se observan los niveles de inversión en Ciencia, Tecnología e Innovación, se evidencia la brecha que existe entre países competitivos y generadores de nuevos conocimientos como Estados Unidos, los miembros de la OCDE, la Unión Europea y China, en comparación con países como Chile y Colombia.

Gráfico 3. Inversión en Ciencia, Tecnología e Innovación como porcentaje del PIB

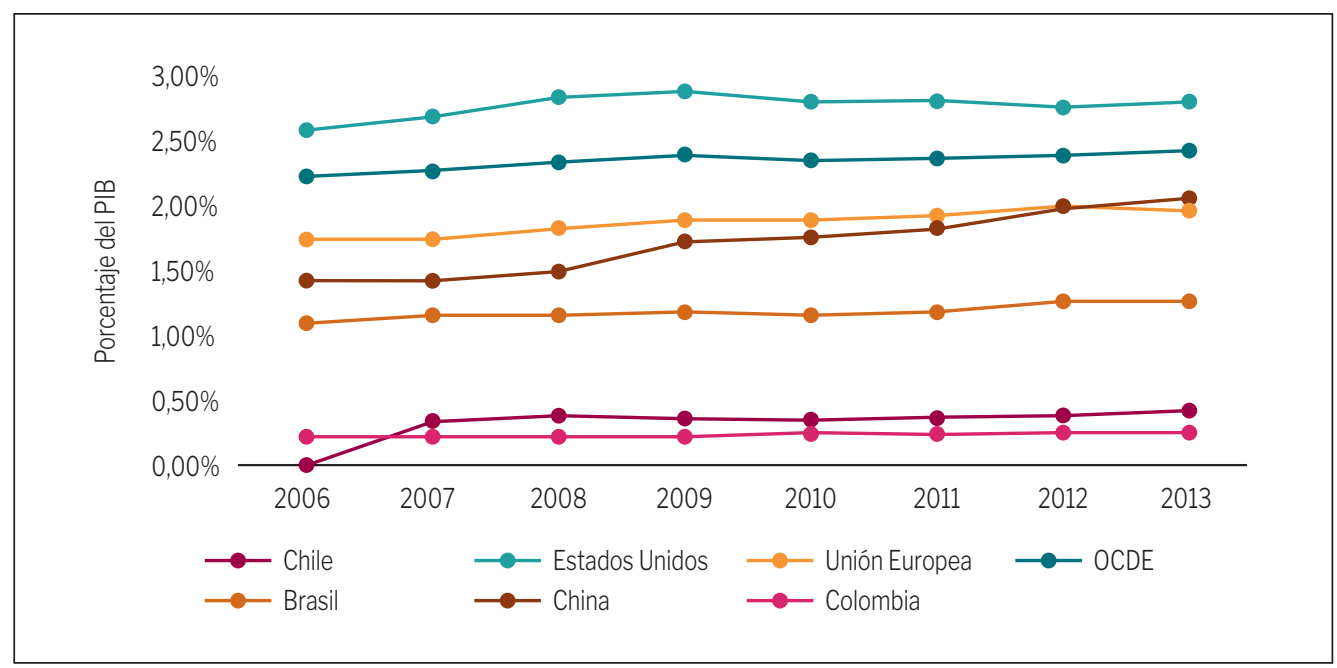

Fuente: elaboración propia con base en las estadísticas de la OCDE (2018). 
Por esta razón, es comprensible que países como Colombia no incrementen los procesos de generación de conocimiento, cuando los niveles de inversión y gasto son pequeños en comparación con otros países, y cuando el mercado laboral no reconoce o diferencia de forma significativa la formación académica como factor de reconocimiento salarial. Lo importante es, ahora, cambiar esa tendencia.

\section{Procesos de enseñanza y aprendizaje: el desafío de la educación superior}

Los principales indicadores de la educación muestran que Colombia ha logrado avanzar en términos de cobertura, hasta alcanzar niveles de participación estudiantil que están por encima del promedio de los países de la OCDE. Ahora, de acuerdo con los pilares fundamentales plateados por la Unesco, el camino por recorrer aún es largo.

Por un lado, las instituciones universitarias y las empresas van por rumbos diferentes, al punto en que para los empleadores el título universitario no se constituye en un aspecto diferenciador de la fuerza de trabajo. En gran parte, esto se debe a que las empresas comprenden la importancia de la formación profesional, pero no necesariamente de la educación.

En este sentido, el gobierno nacional ha establecido como prioridad las políticas resumidas en la tabla 6 para mejorar la educación superior.

Tabla 6. Recomendaciones de política nacional para la educación superior

\begin{tabular}{|c|c|c|}
\hline Política & Aspectos fundamentales & Recomendaciones \\
\hline 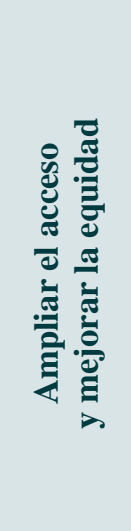 & $\begin{array}{l}\text { - Ayudar a los estudiantes a } \\
\text { tomar decisiones informadas. } \\
\text { Reducir las tasas de deserción } \\
\text { y mejorar la retención y la } \\
\text { finalización de estudios. } \\
\text { - Fortalecer el apoyo financiero } \\
\text { para los estudiantes de bajos } \\
\text { ingresos. } \\
\text { Mejorar la equidad entre } \\
\text { regiones. }\end{array}$ & $\begin{array}{l}\text { - } \quad \text { Garantizar la accesibilidad de } \\
\text { la información para apoyar las } \\
\text { decisiones de los estudiantes. } \\
\text { - Enfocar el apoyo académico } \\
\text { sobre los estudiantes en ries- } \\
\text { go. } \\
\text { - } \text { Eliminar las barreras finan- } \\
\text { cieras de la matrícula de estu- } \\
\text { diantes de bajos ingresos. } \\
\text { La equidad regional debe en- } \\
\text { cabezar las reformas. }\end{array}$ \\
\hline
\end{tabular}




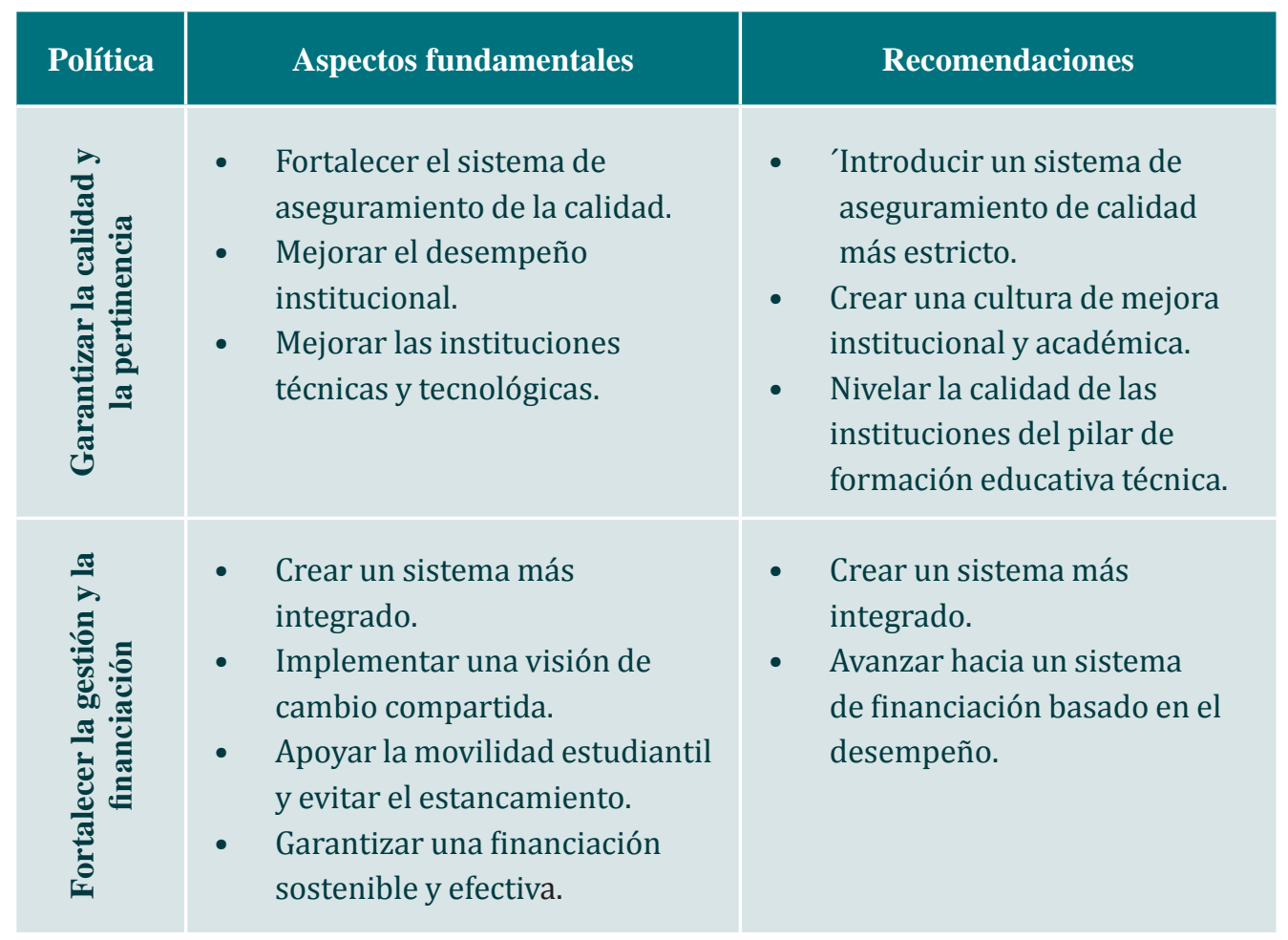

Fuente: Elaboración propia con base en el informe de la OCDE (2014).

Ahora, según la Organización Internacional del Trabajo, OIT, en la Recomendación 150 sobre desarrollo de los recursos humanos de 1975, y en su adopción de 2004, se estipula que la formación profesional es una actividad cuyo objeto es descubrir y desarrollar las aptitudes humanas para una vida activa, productiva y satisfactoria, y esta formación es necesaria, tanto como la educación, para que una persona pueda ser empleable. En este sentido, de acuerdo con Casanova (2003):

Se puede afirmar que la formación profesional es simultáneamente tres cosas:

- Es una actividad de tipo educativo, que se orienta a proporcionar los conocimientos, habilidades y destrezas necesarios para desempeñarse en el mercado de trabajo, sea en un puesto determinado, una ocupación o un área profesional...

- Es una actividad vinculada a los procesos de transferencia, innovación y desarrollo de tecnología. La propia transmisión de conocimientos, habilidades y destrezas implica de por sí un tipo de transferencia tecnológica a los trabajadores y, a través de ellos, a las empresas...

- La formación profesional es un hecho laboral y, como tal, posee un lugar indiscutible dentro de las relaciones de trabajo. Ella concita el interés creciente de gobiernos, 
empresarios y trabajadores, en la medida que se percibe cada vez con mayor claridad la importancia de su aporte a la distribución de las oportunidades de empleo y de trabajo en general, a la elevación de productividad y la mejora de calidad y la competitividad, al logro de condiciones apropiadas y saludables de trabajo, así como en su potencial como espacio de diálogo social a diversos niveles. (p. 10)

Entonces, si la formación profesional es un espacio que las instituciones no educativas pueden atender y es suficiente para satisfacer las necesidades del mercado laboral, el rol principal de la universidad se pierde y pertenecer o no a un sistema educativo de tercer nivel se vuelve obsoleto para la sociedad. Por lo tanto, las instituciones de educación superior dejarían de ser pertinentes si no comienzan a marcar un espacio diferenciador.

Así, para poder generar un espacio diferenciador, es imperativo que las universidades creen espacios para vincularse con la sociedad y comprender las necesidades y retos a los cuales se enfrentan, asegurando el proceso de interrelación ineludible para el funcionamiento de un sistema. Este espacio diferenciador solo se podrá dar en la medida en que la investigación, la enseñanza y el aprendizaje puedan responder los retos del mundo actual.

En este sentido, la característica fundamental del mundo de hoy es la incorporación de los procesos tecnológicos dentro de las actividades del día a día, la adaptación a la era digital. Esta apropiación de la tecnología implica el alcance de la calidad, pues según Yanes (sf), genera impactos en la sociedad a través de:

- Los medios de información, pues en función del conocimiento previo que tengan los individuos respecto a una información tendrán capacidad de juicio para interpretar la información suministrada.

- La educación, porque la tecnología puede facilitar el trabajo interdisciplinar y mejorar la interacción entre el contexto real de la sociedad y los procesos de enseñanza y aprendizaje de las instituciones de educación superior, quienes deben intervenir de forma innovadora (no tradicional o magistral), en cada uno de estos escenarios.

- La gestión del conocimiento, en la medida en que se reconozca que la forma en la cual aprendemos ha cambiado y evolucionado con el uso de las herramientas tecnológicas.

- La formación del capital intelectual, que cambian en la medida que las interacciones se transforman y requieren de una relación cada vez más estrecha entre la sociedad y las instituciones de educación superior.

Adicionalmente, el uso de estas herramientas tecnológicas y su incorporación dentro de las actividades educativas facilitará los procesos de internacionalización de los conocimientos en menor tiempo y con menor costo. Esto se hará de forma más eficiente en la medida en que los grupos académicos de investigación y los estudiantes universitarios participen activamente en la interrelación con otros miembros de la comunidad académica extranjera. 
Considerando todo lo anterior, el éxito de las políticas planteadas dependerá de la forma en que se enfrenten los nuevos desafíos tecnológicos, por lo que transformar los procesos de enseñanza y aprendizaje es un reto fundamental.

\section{Conclusión}

En este capítulo se demostró que abundan los retos para la educación superior en el contexto contemporáneo, y que uno de los más importantes es generar vínculos con el sector productivo para garantizar la pertinencia.

Además, con el reciente ingreso de Colombia a la OCDE se han revelado las principales falencias de la educación superior en el país, y se pone en evidencia que la capacidad para la generación de innovaciones es precaria si se tiene en cuenta la baja participación del gasto en ciencia y tecnología sobre el gasto total.

Otro elemento importante es que los mercados de trabajo no reconocen la importancia de la profesionalización, y los retornos derivados de una inversión en educación superior en Colombia podrían ser inferiores a los que se obtienen en países europeos o en Estados Unidos.

Los hallazgos de este capítulo indican que las universidades no pueden perder el rol activo en la construcción de sociedad, y que la conservación de su posición en el orden social depende de forma determinante de procesos de reingeniería que se piensen una educación que responda a las necesidades del contexto.

\section{Referencias}

Carrera, A., Suayter, I. (sf). Origen y evolución de la educación superior. Trabajo presentado en el V Encuentro Nacional y II Latinoamericano, La Universidad como objeto de investigación de la Universidad Nacional, Buenos Aires, Argentina.

Casanova, F. (2003). Formación profesional y relaciones laborales. Montevideo: Ediciones Cinterfor. Recuperado de: https://www.oei.es/historico/etp/formacion_profesional_relaciones_laborales.pdf

Moreno B. J., Ruiz N. P. (2009). La educación superior y el desarrollo económico en América Latina. Publicación de las Naciones Unidas. México: CEPAL.

MEN. (2015). Bases para la Construcción de los Lineamientos de Política Pública del Sistema Nacional de Educación Terciara (SNET), Bogotá: Viceministerio de Educación Superior, Dirección de Fomento de la Educación Superior, Ministerio de Educación Nacional 
OCDE. (2014). Education at a glance 2014: OECD Indicators. París: Publicaciones OCDE. Recuperado de: http://dx.doi.org/10.1787/eag-2014-en

Organización Internacional del Trabajo, (OIT). (1975). R-150. Recomendación sobre desarrollo de los recursos humanos. Recuperado de https://www.ilo.org/dyn/normlex/ es/f?p=1000:12100:9580486219461::NO::P12100_SHOW_TEXT:Y:

Organización Internacional del Trabajo, (OIT). (2004). R-1195. Recomendación sobre desarrollo de los recursos humanos. Recuperado de https://www.ilo.org/dyn/normlex/es/ f?p=1000:12100:9580486219461:12100:NO::P12100_INSTRUMENT_ID:312533:

Unesco. (1995). Documento de Política para el cambio y el desarrollo de la Educación Superior. Francia: Unesco.

Yenes G., J. (sf). Las TIC y las crisis de la educación: algunas claves para su comprensión. Biblioteca Digital Virtual Educa: OEA. 